\title{
Case fatality of myocardial infarction among shift workers
}

\author{
Jonas Hermansson • Katja Gillander Gådin • Berndt Karlsson • \\ Christina Reuterwall $\cdot$ Johan Hallqvist $\cdot$ Anders Knutsson
}

Received: 6 March 2014 / Accepted: 16 September 2014 / Published online: 27 September 2014

(C) Springer-Verlag Berlin Heidelberg 2014

\begin{abstract}
Purpose Shift work has been associated with an excess risk of cardiovascular disease (CVD) and more specifically myocardial infarction (MI). The majority of the studies that found a positive association between shift work and CVD have been based on incidence data. The results from studies on cardiovascular-related mortality among shift workers have shown little or no elevated mortality associated with shift work. None of the previous studies have analysed short-term mortality (case fatality) after MI. Therefore, we investigated whether shift work is associated with increased case fatality after MI compared with day workers.

Methods Data on incident cases with first MI were obtained from case-control study conducted in two geographical sites in Sweden (Stockholm Heart Epidemiology Program and Västernorrland Heart Epidemiology Program), including 1,542 cases (1,147 men and 395 women) of MI with complete working time information and 65 years or younger. Case fatality was defined as death within 28 days of onset of MI. Risk estimates were calculated using logistic regression.

Results The crude odds ratios for case fatality among male shift workers were 1.63 [95\% confidence interval
\end{abstract}

J. Hermansson $(\triangle) \cdot$ K. Gillander Gådin · A. Knutsson Department of Health Sciences, Mid Sweden University, 85170 Sundsvall, Sweden

e-mail: jonas.hermansson@sll.se

B. Karlsson · C. Reuterwall

Department of Public Health and Clinical Medicine, Occupational Medicine, University of Umeå,

90187 Umeå, Sweden

J. Hallqvist

Department of Public Health and Caring Sciences, Preventive

Medicine, Uppsala University, Box 564, 75122 Uppsala, Sweden
(CI) $1.12,2.38]$ and 0.56 (95\% CI $0.26,1.18)$ for female shift workers compared with day workers. Adjustments for established cardiovascular risk factors such as diabetes type II and socio-economic status did not alter the results.

Conclusion Shift work was associated with increased risk of case fatality among male shift workers after the first MI.

Keywords Cardiovascular disease - Case-control .

Shift work $\cdot$ Risk estimation $\cdot$ Epidemiology

\section{Introduction}

A growing number of studies from several countries have shown that shift work is associated with an increased risk of coronary heart disease (CHD), especially myocardial infarction (MI) (Fujino et al. 2006; Knutsson 1989; Tenkanen et al. 1997). Numerous hypotheses have been presented in order to explain possible causal mechanisms between shift work and cardiovascular disease (CVD) or CHD. The hypotheses include disruption of the circadian rhythm, sleep deprivation, social stress related to isolation stemming from the divergent working hours, behavioural factors such as smoking, lack of physical activity and poor dietary choices, and low socio-economic status (Bøggild and Knutsson 1999; Frost et al. 2009). Shift work has also been associated with atherosclerosis (Haupt et al. 2008; Puttonen et al. 2009), electrocardiographic changes (Meloni et al. 2013), and arrhythmias (Härenstam et al. 1987).

While the majority of studies that analysed the association between incidence of CVD and shift work was able to confirm such association (Ellingsen et al. 2007; Haupt et al. 2008; Vyas et al. 2012), most studies analysing the association between total mortality of CVD and shift workers have not reported any such association (Bøggild and Knutsson 
1999; Karlsson et al. 2005; Taylor et al. 1972; Vyas et al. 2012). No studies have been focused on the short-term mortality (case fatality) after MI among shift workers. If there is an association between case fatality of MI and shift work, this could represent an important clue in understanding whether shift work is associated with increased CVD mortality. Therefore, the aim of this study was to analyse whether shift work is associated with increased case fatality of MI.

\section{Methods}

Setting and study design

Cases were defined as all first episodes of non-fatal and fatal events of MI and were collected from a previous casereferent database that consisted of one study in two different geographical areas in Sweden [the Stockholm Heart Epidemiology Programme (SHEEP) and the Västernorrland Heart Epidemiology Program (VHEEP)]. The geographical sites had slightly different periods of case identification and age span of the participants, as described below. The analyses in this study were performed on all available data from the study. The combined study base contained all Swedish citizens living in the counties of Stockholm and Västernorrland, respectively, who were free of previous clinically diagnosed MI. In Stockholm County, all male cases were identified during a 2-year period (1992-1993) and all female cases during a 3-year period (1992-1994). During the period from January to October 1992, the age span was 45-65 years, but from November 1, 1992 and onwards the upper age limit was 70 years. In Västernorrland County, the age span was restricted to 45-65 years, and cases were identified during the period between March 1993 and March 1995.

\section{Participants}

MI cases were included at the time of disease incidence. They were identified from (a) the coronary and intensive care units at the internal medicine departments at all the emergency hospitals within the counties of Stockholm and Västernorrland, (b) the hospital discharge register for the same counties, and (c) death certificates from the National Register of Causes of Death at Statistics Sweden. The criteria of diagnosis included (a) certain symptoms according to information on case history, (b) specified changes in blood concentrations of the enzymes creatine kinase and lactate dehydrogenase, (c) specified electrocardiographic changes, and (d) necropsy findings. The diagnosis of MI required two of the criteria $\mathrm{a}-\mathrm{c}$ to be met, or that necropsy findings showed myocardial necrosis of an age compatible with the time of onset of the disease. A group of cardiologists assessed the medical inclusion and exclusion criteria for hospital cases. The same diagnostic criteria were applied for all cases.

Statistics Sweden identified patients who died before admission to hospitals. Among these cases, $72 \%$ were autopsied and had signs of recent MI. For the remaining $28 \%$ of cases in this group, the physician issued the death certificate based on the diagnosis of information on disease history and death circumstances.

All cases were checked for previous hospital admissions for MI from 1971 in the Stockholm heart epidemiology programme and from 1985 in the Västernorrland infarction programme in the hospital discharge register. If such an episode was found, the person was excluded from the study base. Participants with previous infarctions [consistent with diagnosis code 412 in international classification of diseased ninth revision (ICD-9)], as judged from necropsy findings only, were not excluded from the study base. Non-fatal cases answered a questionnaire as soon as possible after recovering. For fatal cases, questionnaire information was obtained from a close relative 3-6 month after the occurrence of MI. The study has previously been described in detail (Reuterwall et al. 1999).

The data in the present study consisted of 1,961 cases of MI, and 1,542 of these cases had complete working time information and were subsequently used in the regression analyses. Among male cases, $18.3 \%$ were shift workers and $81.7 \%$ were day workers (210/937). Among females, the corresponding numbers were $17.5 \%$ and $82.5 \%$ (69/326). Case fatality in this study was the proportion of cases with the first time MI consistent with the previously described diagnostic criteria, which was fatal within 28 days after the event. The participants had a mean age of 57.11 (SD 5.77, median 58) years.

Exposure information

\section{Shift work}

Shift work exposure was assessed through the following questions: (1) Did you undertake shift work (during the most recent 5 years of work)? If the answer was yes, the respondent was asked to add information about the type of shift schedule. (2) To reduce the risk of including participants that were retired, the age limit for inclusion was set to 65 years, and unemployed or retired cases were excluded. The second question concerned when during the day that the majority of the work hours was scheduled. The response alternatives were (a) 06:00-18:00, (b) 18:0022:00, (c) 22:00-06:00, (d) a combination of (a) and (b), (e) a combination of (b) and (c), (f) a combination of (a) and (c), and (g) a combination of (a), (b), and (c). If a person 
answered yes to question 1 or chose response alternatives b-f to question 2, he or she was regarded as being exposed to shift work $(14.2 \%)$. If the respondent answered no to question 1 and did not chose alternatives $\mathrm{b}-\mathrm{f}$ of question 2 , the respondent was regarded as day worker $(85.7 \%)$, and only $0.2 \%$ of the respondents were excluded because their answers were not possible to categorise. In total, 270 males and 194 females were excluded based on them being 65 years or older at the time of their MI. None of the excluded cases were over 70 years of age. Shift workers were coded 1 , and day workers were coded 0 .

\section{Physical inactivity}

Respondents that had answered that they never or only occasionally were physical active in their spare time were considered physical inactive, while those who did not meet this criteria were considered physically active and were used as reference.

\section{Tobacco smoking}

Smoking habits were divided into current smokers and nonsmokers. Current smokers included ex-smokers who had stopped smoking within the last 2 years before inclusion, and non-smokers included ex-smokers quitting more than 2 years ago.

\section{Elevated BMI}

Height and weight measures were taken at the physical examination. For those who failed to attend the physical examination, but who had answered the questionnaire (3.8\% of the population with complete BMI data), the reported height and weight were used in order to calculate BMI. A BMI equal to or exceeding $28 \mathrm{~kg} / \mathrm{m}^{2}$ was considered high.

\section{Diabetes type II}

Diabetes type II was considered present if a participant had a level of fasting glucose above $6.7 \mathrm{mmol} / \mathrm{L}$ or if the question of known diabetes type II was reported in the questionnaire. It was also considered present if a participant required insulin or if a participant regularly medicated for disease consistent with ICD 9-code 250 or if a participant reported pharmacological treatment against diabetes type II in the questionnaire.

\section{Low socio-economic status}

Educational level was used as a proxy variable to estimate socio-economic status. Cases with compulsory school
(9 years) as their highest education were considered to have low socio-economic status; participants with higher education were used as reference.

\section{Elevated blood pressure}

High blood pressure was considered present if a respondent had either $\geq 170 \mathrm{mmHg}$ in systolic or $\geq 95 \mathrm{mmHg}$ in diastolic blood pressure, or reported pharmacological treatment for disease consistent with ICD 9-code 40, or earlier pharmacological treatment for hypertension that ended $<5$ years before inclusion in the database.

\section{Job strain}

Job strain was measured by using a version of the questionnaire in Swedish (Theorell et al. 1988). The questionnaire consisted of five questions about psychological demands and six questions about decision latitude, each question with four response alternatives ranging from $1=$ never to $4=$ always or almost always. The work experience during the last 5 years before inclusion in the study was considered. The variable was based on the individual quota between demand and control. Job strain was considered present for respondents who were in the upper tertile in the demand questions and the lower tertile in the decision latitude. Participants with job strain were coded 1 , and the others were coded 0 .

Statistical analysis

All calculations were made separately for men and for women. Case fatality rates (the percentage of fatalities of MI within 28 days after the event) were calculated for shift and day workers. chi-square analyses were used on dichotomous variables to analyse differences between shift workers and day workers in exposure characteristics, and independent samples $t$ test were used to analyse differences in average age. A $p$ value of 0.05 or less was considered statistically significant.

Unconditional logistic regression was used since conditional logistic regression produced similar results, to estimate the odds ratio (OR) and $95 \%$ confidence interval (95\% CI), as a measure of shift work's association on case fatality. Logistic regression analyses were conducted for each exposure characteristic. Included exposure characteristics were physical inactivity, tobacco smoking, elevated BMI, diabetes type II, low socio-economic status, elevated blood pressure, and job strain. Exposure characteristics were selected based on their previous inclusion in studies analysing case fatality of MI (Wannamethee et al. 1995; Yang et al. 2012), and for job strain based on the association with incidence of CHD (Kuper and Marmot 2003). In 
Table 1 Characteristics (\%) of day and shift worker with MI

Results of chi-square analysis. A total of 210 male shift workers and 937 male day workers, and 69 female shift workers and 326 female day workers were included in the analysis

\begin{tabular}{|c|c|c|c|c|c|c|}
\hline & \multicolumn{3}{|l|}{ Men } & \multicolumn{3}{|l|}{ Women } \\
\hline & Day work & Shift work & $p$ value & Day work & Shift work & $p$ value \\
\hline Physical inactivity & 36.8 & 42.1 & 0.047 & 44.5 & 41.1 & 0.47 \\
\hline Current smoking & 39.3 & 52.2 & $<0.001$ & 39.4 & 55.0 & $<0.001$ \\
\hline $\mathrm{BMI} \geq 28$ & 26.1 & 33.3 & 0.003 & 28.3 & 43.2 & $<0.001$ \\
\hline Diabetes type II & 11.5 & 16.2 & 0.008 & 10.8 & 14.0 & 0.29 \\
\hline Low socio-economic status & 20.5 & 52.7 & $<0.001$ & 34.9 & 72.0 & $<0.001$ \\
\hline Elevated blood pressure & 27.0 & 28.9 & 0.43 & 38.2 & 53.7 & 0.64 \\
\hline Job strain & 23.4 & 36.3 & $<0.001$ & 29.6 & 27.6 & $<0.001$ \\
\hline
\end{tabular}

order to justify the inclusion, given that the area of case fatality of MI and its association with shift work is not well studied, we analysed the relative change in estimated effect size after adjustment of the crude OR for each exposure characteristic on its own (Rothman et al. 2008). This method is known as change-in-estimate or non-collapsibility, and states that a change in relative estimated effect is assessed via the regression coefficient. In our study, changes of the regression coefficient exceeding $\pm 10 \%$ were selected as the cut-off level (Greenland 1989), such change indicates that the exposure characteristics might be a confounder (Rothman et al. 2008). Two different multivariate logistic regression analyses were performed, one with adjustments for biologically related covariates and the other one with behaviourally related covariates. Moreover, the regression analyses were adjusted for age as a continuous variable. The statistical analyses were carried out using SPSS 20.0 for PC.

\section{Results}

The case fatality of MI among male shift workers was $21.2 \%$ of 210 cases, compared with $14.3 \%$ of 937 cases among day workers. The case fatality for female shift workers was $13 \%$ of 69 cases of compared with $21.2 \%$ of 326 cases among female day workers.

Of the included MI cases, $80.7 \%$ were from SHEEP and $19.3 \%$ from VHEEP. Additional analyses comparing case fatality among males against females did not show that male gender was associated with an increased risk of case fatality in general or when comparing male to female shift workers (results not shown). Moreover, additional analyses showed no difference between males and females in age (results not shown).

Table 1 shows that shift workers were more exposed to current smoking, BMI equal to or above 28 , low socioeconomic status, and job strain for both men and women. Physical inactivity and diagnosed diabetes type II were more prevalent among male shift workers compared with male day workers, but no such difference was seen for women. Female day workers were more exposed to job strain compared with female shift workers. No other differences were found.

Table 2 shows that the crude OR for case fatality following an MI for male shift workers was 1.63 (95\% CI 1.122.38). Adjustments for potential risk factors yielded only minor changes in the OR except for low socio-economic status. When combining elevated BMI, diabetes type II, and elevated blood pressure in a multivariate regression model to analyse the associations related to medical risk factors, the OR for male shift workers was 2.17 (95\% CI 1.46$3.23)$. In the adjustments for elevated blood pressure and the first multivariate adjustment, more day workers than shift workers were included as cases (results not shown). In the multivariate regression model combining risk factors more directly associated with behavioural and social factors, the OR from male shift workers is relatively constant compared with previous results reported in Table 2.

The OR for female shift workers was 0.56 (95\% CI 0.26-1.18). Adjustment for the potential risk factors in both univariate and multivariate regression models did not yield any changes to the results.

Socio-economic status was also analysed using occupational status as a proxy variable comparing blue-collar workers with white-collar workers. The results for both male and female shift workers were similar to the results using educational level as proxy variable (results not shown). Similar regressions as presented here were also performed for the entire data set (including participants over 65 years). The results were similar to the ones presented above with the difference that the OR for males were in general about $20 \%$ lower (results not shown).

In addition, we performed multivariate regression models containing all included covariates and then removing them step-by-step. The OR for shift workers ranged from 1.82 to 1.87 for males and from 0.29 to 0.48 for females. None of the results for female shift workers were significant.

In order to test potential confounder effects, we analysed the change in the regression coefficient in the regression analysis when removing each covariate. No changes over $\pm 10 \%$ in the regression coefficient were noted for male or female shift workers. 
Table 2 OR of association between case fatalities after first event of MI and shift work for men and women and adjusted for possible confounding

\begin{tabular}{|c|c|c|c|c|}
\hline & \multicolumn{2}{|l|}{ Men } & \multicolumn{2}{|c|}{ Women } \\
\hline & OR & $95 \% \mathrm{CI}$ & OR & $95 \% \mathrm{CI}$ \\
\hline Shift work & 1.63 & $1.12,2.38$ & 0.56 & $0.26,1.18$ \\
\hline Shift work, adjusted for physical inactivity & 1.54 & $1.05,2.27$ & 0.66 & $0.3,1.43$ \\
\hline Shift work, adjusted for current smoking & 1.65 & $1.13,2.41$ & 0.55 & $0.26,1.16$ \\
\hline Shift work, adjusted for BMI $\geq 28$ & 1.59 & $1.07,2.38$ & 0.4 & $0.15,1.05$ \\
\hline Shift work, adjusted for diabetes type II & 1.67 & $1.14,2.44$ & 0.57 & $0.26,1.2$ \\
\hline Shift work, adjusted for low socio-economic status & 1.38 & $0.82,2.32$ & 0.31 & $0.1,1.05$ \\
\hline Shift work, adjusted for elevated blood pressure & 1.82 & $1.22,2.7$ & 0.47 & $0.21,1.1$ \\
\hline Shift work, adjusted for job strain & 1.62 & $1.1,2.39$ & 0.41 & $0.17,1.0$ \\
\hline Shift work, adjusted for BMI $\geq 28$, diabetes type II, and elevated blood pressure & 2.17 & $1.46,3.23$ & 0.47 & $0.18,1.35$ \\
\hline $\begin{array}{l}\text { Shift work, adjusted for physical inactivity, current smoking, low socio-economic } \\
\text { status, and job strain }\end{array}$ & 1.65 & $1.05,2.6$ & 0.38 & $0.11,1.29$ \\
\hline
\end{tabular}

Results of age-adjusted logistic regression analysis with $95 \%$ CI. A total of 210 male shift workers and 937 male day workers, and 69 female shift workers and 326 female day workers were included in the analysis

\section{Discussion}

We found an association between increased risks of case fatality within 28 days after MI among male shift workers. When adjusting for potential confounding variables in different regression models, the OR changed marginally. No such association was found for female shift workers. The low number of female shift workers in the analysis with associated low power issue could partly explain this.

Other studies have identified physical activity as a protective factor against all-cause mortality and CVD mortality (Andersen et al. 2000) and case fatality of MI (Wannamethee et al. 1995). After adjusting for physical inactivity, the OR decreased for male shift workers. This result may be explained by the fact that the prevalence of physical inactivity was higher among male shift workers compared with day workers. No changes in OR were found among women when adjusting for physical inactivity.

Adjusting for current tobacco smoking, BMI $\geq 28$, or diabetes type II did not result in any major change in the OR for shift workers. Socio-economic status has been identified earlier as a risk factor for case fatality (Rosvall et al. 2008; Salomaa et al. 2001). We found that low socio-economic status was more common among male shift workers compared with male day workers. In our study, we found no greater OR changes when adjusting for socio-economic status for increased case fatality for male or female shift workers.

Adjusting for elevated blood pressure for male shift workers gave a marked increase in the OR for case fatality in this study. It is likely that the increase in OR relates to the higher prevalence of elevated blood pressure among fatal day-working cases. Adjusting for job strain did not lead to any OR change for male or female shift workers in the analyses. This is interesting given that job strain was more prevalent among male shift workers and that recent findings have associated job strain with not only increased risk of incident CHD but also with increased inflammatory burden (Emeny et al. 2013). This could have meant that shift workers affected by job strain could also be at greater risk of case fatality after MI, but the results did not support that assumption.

The multivariate regression analyses used to assess the effects of combined risk factors, and their association with case fatality identified an elevated OR for male shift workers and biological risk factors. This was likely related to the higher prevalence of male day workers with the given characteristics. This may be explained by the fact that the included risk factors are associated with a poorer prognosis after an MI.

For further studies of case fatality among shift workers, it would be of interest to study a number of factors that could hold part of the explanation behind the results from this study. For example, if the arteries of shift workers are more affected by atherosclerosis (Haupt et al. 2008), it is likely that this would have weakened the intima of the blood vessel and thereby increasing the risk of rupture and thrombosis occurring when the patient is vulnerable because of the previous MI. Shift work has also been associated with arrhythmias (Härenstam et al. 1987), prolonged QTc interval, and conduction disorders (Meloni et al. 2013), which in term may indicate a poorer prognosis after a MI. That may also play a role in the increased association between case fatalities of MI for male shift workers reported in this study and would be of interest for further studies. 
It would also be of interest to perform similar analyses on newer study materials as there are studies showing that case fatality in the general population has declined since the material for this study was gathered (Mannsverk et al. 2012). There are differences in the treatment of modern MI patients compared with the ones included in this study; the amount of patients undergoing revascularisation is higher today, and the same goes for having beta-blockers, aspirin, or statins at discharge from hospital (Mannsverk et al. 2012).

The result that female shift workers not had an increased risk for case fatality compared with female day workers needs to be elaborated in future studies. The labour market is gender segregated in Sweden with more women in, e.g. the health care sector and more men in, e.g. the transport sector (Statistics Sweden 2012), and that means that the working situation for women and men in shift work compared with day work could be different.

This study reports a case fatality among male shift workers of $21.2 \%$ and male day workers of $14.3 \%$. For females, the corresponding case fatality for shift workers was $13 \%$ and for day workers $21.2 \%$. Male shift workers and female day workers in this study have relatively similar case fatality compared to other Swedish reports, while the case fatality for male day workers and female shift workers are lower in comparison (Rosengren et al. 2001). We have not found any recently published studies discussing case fatality of MI and shift work. There has been a trend in Sweden of decreasing case fatality rates after first time MI from 1978 to 2008, for both men and women (Yang et al. 2012). If this study would be repeated using a dataset reflecting the working conditions in Sweden 2014, the results would likely indicate lower case fatality rates for both shift workers and day workers. However, this study investigated the relative difference between shift workers and day workers regarding case fatality after MI. Therefore, the relative difference in case fatality risk of MI reported for male shift workers compared with male day workers in this study is likely to be relevant as of today even though the absolute risk has decreased.

As previously noted, many of the studies conducted on the association between CHD mortality and shift work are subjected to results potentially influenced by confounding factors (Frost et al. 2009). As reported in the results of our analysis, we found no results supporting confounding effects of the included covariates in our analysis. The findings of this study show that male shift workers may have an increased risk of case fatality after MI, and this contradicts the findings of the meta-analysis published in 2012 (Vyas et al. 2012). This may be explained by the fact that this study analyses males and females separately, while the meta-analysis did not report gender-specific mortality or case fatality.
Blood cholesterol and triglycerides were not assessed in this analysis due to lack of such data for diseased cases. There is a potential risk of misclassification regarding the use of questionnaire data for deceased cases submitted by a close relative, primarily due to recall bias. This fact might explain our result to some extent, but because no studies were found that discussed gender differences in recall bias or recall bias related to shift work variables reported by relatives, the level of impact on the results remains hypothetical.

Shift work is often defined as a work schedule including work hours outside the daytime schedule. A limitation in epidemiological studies is that the information on shift work exposure is often drawn from questionnaire data (Wang et al. 2011). The questions used to determine that shift work exposure varies from rather generic questions without attempts of determining vital components of the shift work exposure (Costa 2003) to more intricate questions that deal with type of shift work and exposure duration. The questionnaire data regarding working time conditions that formed the basis of the shift work variable in this study and the definition of shift- and day work derived from that question have been used in previous studies (Knutsson et al. 1999). The definition used in this study is lacking information on lifetime exposure to shift work, representing a limitation in this study. The definition of shift work in this study was based on questionnaire data concerning the last 5 years of work. This creates the potential risk of misclassification; there is a risk of respondents having trouble in correctly determining their working conditions. This may perhaps lead to an underestimation or an overestimation of the risk associated with shift work itself, but it has been proposed that questionnaire data on shift work would be less subjected to recall bias (Kolstad 2008).

To our knowledge, this study is the first to analyse the association between case fatality of MI and shift work. Therefore, the findings of this study are to be regarded as preliminary, and more research in the field is needed.

\section{Conclusion}

This study shows that male shift workers have an increased association with case fatality after MI. More studies are needed to further clarify whether the same association exists for female shift workers. In all, male shift workers association with increased case fatality after MI should be addressed in preventive efforts in order to decrease suffering.

Conflict of interest The authors declare that they have no conflict of interest. 


\section{References}

Andersen LB, Schnohr P, Schroll M, Hein HO (2000) All-cause mortality associated with physical activity during leisure time, work, sports, and cycling to work. Arch Intern Med 160:1621-1628

Bøggild H, Knutsson A (1999) Shift work, risk factors and cardiovascular disease. Scand J Work Environ Health 25:85-99

Costa G (2003) Shift work and occupational medicine: an overview. Occup Med 53:83-88

Ellingsen T, Bener A, Gehani AA (2007) Study of shift work and risk of coronary events. J R Soc Promot Health 127:265-267

Emeny RT, Zierer A, Lacruz ME et al (2013) Job strain-associated inflammatory burden and long-term risk of coronary events: findings from the MONICA/KORA Augsburg case-cohort study. Psychosom Med 75:317-325. doi:10.1097/PSY.0b013e3182860d63

Frost P, Kolstad HA, Bonde JP (2009) Shift work and the risk of ischemic heart disease-a systematic review of the epidemiologic evidence. Scand J Work Environ Health 35:163-179

Fujino Y, Iso H, Tamakoshi A et al (2006) A prospective cohort study of shift work and risk of ischemic heart disease in Japanese male workers. Am J Epidemiol 164:128-135. doi:10.1093/aje/kwj185

Greenland S (1989) Modeling and variable selection in epidemiologic analysis. Am J Public Health 79:340-349

Härenstam A, Theorell T, Orth-Gomer K, Palm U, Unden A (1987) Shift work, decision latitude and ventricular ectopic activity: a study of 24-hour electrocardiograms in Swedish prison personnel. Work Stress 1:341-350

Haupt CM, Alte D, Dörr M et al (2008) The relation of exposure to shift work with atherosclerosis and myocardial infarction in a general population. Atherosclerosis 201:205-211. doi:10.1016/j. atherosclerosis.2007.12.059

Karlsson B, Alfredsson L, Knutsson A, Andersson E, Toren K (2005) Total mortality and cause-specific mortality of Swedish shift-and dayworkers in the pulp and paper industry in 1952-2001. Scand J Work Environ Health 31:30-35

Knutsson A (1989) Shift work and coronary heart disease. Scand J Soc Med Suppl 44:1-36

Knutsson A, Hallquist J, Reuterwall C, Theorell T, Akerstedt T (1999) Shiftwork and myocardial infarction: a case-control study. Occup Environ Med 56:46-50

Kolstad HA (2008) Nightshift work and risk of breast cancer and other cancers - a critical review of the epidemiologic evidence. Scand J Work Environ Health 34:5-22

Kuper H, Marmot M (2003) Job strain, job demands, decision latitude, and risk of coronary heart disease within the Whitehall II study. J Epidemiol Community Health 57:147-153

Mannsverk J, Wilsgaard T, Nøjstad I et al (2012) Age and gender differences in incidence and case fatality trends for myocardial infarction: a 30-year follow-up. The Tromso Study. Eur J Prev Cardiol 19:927-934. doi:10.1177/1741826711421081

Meloni M, Setzu D, Del Rio A, Campagna M, Cocco P (2013) QTc interval and electrocardiographic changes by type of shift work. Am J Ind Med 56:1174-1179. doi:10.1002/ajim.22207
Puttonen S, Kivimäki M, Elovainio M et al (2009) Shift work in young adults and carotid artery intima-media thickness: the Cardiovascular Risk in Young Finns study. Atherosclerosis 205:608613. doi:10.1016/j.atherosclerosis.2009.01.016

Reuterwall C, Hallqvist J, Ahlbom A et al (1999) Higher relative, but lower absolute risks of myocardial infarction in women than in men: analysis of some major risk factors in the SHEEP study. The SHEEP Study Group. J Intern Med 246:161-174

Rosengren A, Spetz CL, Koster M, Hammar N, Alfredsson L, Rosen M (2001) Sex differences in survival after myocardial infarction in Sweden; data from the Swedish National Acute Myocardial Infarction Register. Eur Heart J 22:314-322. doi:10.1053/e uhj. 2000.2368

Rosvall M, Gerward S, Engstrom G, Hedblad B (2008) Income and short-term case fatality after myocardial infarction in the whole middle-aged population of Malmo, Sweden. Eur J Pub Health 18:533-538. doi:10.1093/eurpub/ckn059

Rothman K, Greenland S, Lash T (2008) Modern epidemiology. Lippincott Williams \& Wilkins, New York

Salomaa V, Miettinen H, Niemelä M et al (2001) Relation of socioeconomic position to the case fatality, prognosis and treatment of myocardial infarction events; the FINMONICA MI Register Study. J Epidemiol Community Health 55:475-482

Statistics Sweden (2012) Men and women in Sweden. Facts and figures 2012. In: Sweden S (ed). Stockholm. http://www.scb.se/sv_/ Hitta-statistik/Publiceringskalender/Visa-detaljerad-information/? publobjid $=17389+$

Taylor PJ, Pocock SJ, Sergean R (1972) Shift and dayworkers' absence: relationship with some terms and conditons of service. Br J Ind Med 29:338-340

Tenkanen L, Sjoblom T, Kalimo R, Alikoski T, Harma M (1997) Shift work, occupation and coronary heart disease over 6 years of follow-up in the Helsinki Heart Study. Scand J Work Environ Health 23:257-265

Theorell T, Perski A, Åkerstedt T et al (1988) Changes in job strain in relation to changes in physiological state. A longitudinal study. Scand J Work Environ Health 14:189-196

Vyas MV, Garg AX, Iansavichus AV et al (2012) Shift work and vascular events: systematic review and meta-analysis. BMJ 345:e4800. doi:10.1136/bmj.e4800

Wang XS, Armstrong ME, Cairns BJ, Key TJ, Travis RC (2011) Shift work and chronic disease: the epidemiological evidence. Occup Med 61:78-89. doi:10.1093/occmed/kqr001

Wannamethee G, Whincup PH, Shaper AG, Walker M, MacFarlane PW (1995) Factors determining case fatality in myocardial infarction "who dies in a heart attack"? Br Heart J 74:324-331

Yang D, Dzayee DA, Beiki O, de Faire U, Alfredsson L, Moradi T (2012) Incidence and case fatality after day 28 of first time myocardial infarction in Sweden 1987-2008. Eur J Prev Cardiol 19:1304-1315. doi:10.1177/1741826711425340 